\title{
Bibliotecas digitales académicas y web semántica: elementos para la innovación de los servicios bibliotecarios y de información
}

\section{Academic Digital Libraries and Semantic Web: Elements for the Innovation of Library and Information Services}

\author{
Eder Ávila Barrientos* \\ Universidad Nacional Autónoma de México, \\ Facultad de Filosofía y Letras
}

\author{
Recibido: 04 de noviembre 2015 Aceptado: 02 de marzo 2016 \\ Corregido: 07 de marzo 2016 Publicado: 24 de junio 2016
}

\begin{abstract}
Resumen
La biblioteca digital académica es un sistema que ha sido desarrollado mediante el uso de las tecnologías de la información y la comunicación. Su origen se remonta a proyectos de investigación en diversas universidades e instituciones académicas alrededor del mundo. Como parte de su desarrollo evolutivo, en la actualidad se encuentra inmersa en el contexto digital de la web semántica, una web extendida y dotada de mayor significado, apoyada en lenguajes universales, lo cual va a permitir que los usuarios puedan encontrar respuestas a sus preguntas de forma más eficiente y efectiva, gracias al establecimiento de relaciones significativas de información. El objetivo de este trabajo es analizar la presencia de las bibliotecas digitales académicas en la web semántica, para identificar los elementos que propicien la innovación en sus servicios bibliotecarios y de información. Apoyados en el análisis documental, la hermenéutica y el análisis del discurso, se localizan y estudian diversos recursos de información de la literatura especializada en Bibliotecología y Estudios de la Información que abordan los objetos de estudio presentados en este trabajo.
\end{abstract}

Palabras clave

Bibliotecas digitales académicas, web semántica, servicios bibliotecarios y de información

\begin{abstract}
The academic digital library is a system that has been developed through the use of information and communication technologies. Its origin goes back to research projects at various universities and academic institutions around the world. As part of their evolutionary development, now is immersed in the digital context of the semantic web, a web extended and equipped with greater meaning, based on universal languages, will allow users to find answers to your questions more efficiently and effectively through the establishment of meaningful relationships of information. The objective of this work lies in the presence of digital academic libraries in the semantic web, to identify the elements that foster innovation in library and information services. Supported the documentary analysis and hermeneutic and discourse analysis, they are located and analyze various information resources of literature specializing in library and information studies addressing the objects of study presented in this article.
\end{abstract}

Keywords

Academic Digital Libraries, Semantic Web, Library and Information Services. 


\section{Introducción}

En la última década ha surgido un nuevo reto para las bibliotecas académicas convencionales provocado por el vertiginoso crecimiento de la información digital y la aplicación de las tecnologías de la información y comunicación (TIC) en sus estructuras. Este fenómeno ha motivado la aparición de la biblioteca digital académica (BDA).

Ante ello, los servicios tradicionales de las bibliotecas presentan cambios sustanciales que inciden en forma directa en el modo en que la información se hace accesible al usuario. Los usuarios de la biblioteca académica tradicional utilizan la modalidad digital de esta biblioteca de una manera remota, es decir, desde cualquier ubicación geográfica y espacial del mundo y manifiestan comportamientos informativos novedosos y demandas de información aún más complejas relacionadas con su quehacer académico e investigativo en las instituciones de educación superior.

La presencia de la BDA en el entorno de la web pone de manifiesto una serie de interrogantes relacionadas con el desarrollo evolutivo de dicho espacio, ya que en la actualidad se menciona en la literatura especializada el surgimiento de una web con significado, llamada Web Semántica o Web 3.0. La posibilidad que tienen las bibliotecas de interactuar en este entorno será significativa y prometedora para la creación, adaptación o modificación de servicios bibliotecarios y de información en el entorno digital. En este sentido, la innovación aplicada a dichos servicios puede traer consigo grandes cambios en la manera de concebir a la biblioteca en los entornos académicos y de investigación.

A partir de los argumentos presentados en este trabajo, se intentan responder los siguientes cuestionamientos: ¿Qué es una biblioteca digital académica?, ¿De qué manera puede establecerse una interacción entre las BDA y la web semántica? ¿Las bibliotecas académicas y la web semántica proporcionan elementos significativos para la innovación de los servicios bibliotecarios y de información? El objetivo de este trabajo recae en analizar la presencia de las bibliotecas digitales académicas en la web semántica, para identificar los elementos que propicien la innovación en sus servicios bibliotecarios y de información.

\section{Método}

Para el desarrollo de este trabajo se aplicaron dos métodos a la literatura especializada sobre bibliotecas digitales académicas y web semántica. El primero de ellos es el análisis documental, el cual permitió identificar las relaciones temáticas entre los recursos de información que abordan los dos tópicos principales de la investigación. Por otra parte, también se utilizó la hermenéutica y el análisis del discurso para identificar patrones similares en cuanto a la argumentación expuesta por los diversos estudios analizados. Es importante destacar que los tipos de recursos de información tratados en esta investigación corresponden a artículos de revistas arbitradas y monografías especializadas sobre las temáticas anteriormente mencionadas, con una periodicidad del año 2005 en adelante.

\section{bibliotecas}




\section{Bibliotecas digitales académicas (BDA)}

La BDA es un sistema que ha sido desarrollado mediante el uso de las tecnologías de la información y comunicación. Su origen se remonta a proyectos de investigación efectuados en diversas universidades e instituciones académicas alrededor del mundo. Una biblioteca académica forma parte integral de un colegio, universidad u otra institución de enseñanza en el nivel superior; es administrada principalmente para satisfacer las necesidades de información e investigación de los investigadores, estudiantes y profesores de la institución. Es considerada un servicio básico y trascendental de la institución a la que pertenece por su apoyo en el desarrollo de las funciones fundamentales de educación, docencia, investigación y difusión de la cultura. En términos generales, siguiendo a Torres "una biblioteca digital académica será aquella biblioteca digital que forma parte de una universidad o institución de educación superior, administrada para satisfacer las necesidades de su comunidad" (2008, p. 45).

Tomando en cuenta las generalidades de la conceptualización de la biblioteca digital, en este trabajo se considera la Biblioteca Digital Académica como un sistema de información en línea perteneciente a una institución de educación superior que integra, organiza, administra y preserva colecciones y contenidos digitales; pone a disposición de su comunidad usuaria, servicios y herramientas que propician el acceso y utilización de sus recursos de información.

La BDA es un sistema de información accesible a través de la Web, que forma parte o es patrocinada por una institución académica de nivel superior. En otras palabras, es una entidad que busca satisfacer las necesidades de información de su comunidad académica (una sociedad científica, literaria o artística establecida con autoridad pública y como establecimiento docente, público o privado, de carácter profesional, artístico, técnico, o simplemente práctico).

Al formar parte de una universidad, la BDA sirve a los fines de educación, investigación y difusión de la cultura, que según Ortega y Gasset (2007) son tres: la enseñanza para la formación de profesionales, la investigación (científica y humanística) y la difusión de la cultura (p. 149). De esta forma, las BDA se categorizan en dos grupos, delimitados por los usuarios a los que sirve y las tareas que apoya: aquellas que se enfocan hacia la educación y las que se dirigen al apoyo a la investigación. En México el concepto de biblioteca académica que apoya la investigación, se relaciona más con el de biblioteca especializada.

\section{a. Atributos de la BDA}

El acceso a los servicios y colecciones de las bibliotecas digitales es una meta para las BDA. Las colecciones de una biblioteca digital académica no sustituyen a los documentos tradicionales, sino extienden los recursos digitales que no pueden ser representados o distribuidos en formato impreso, asimismo permiten identificar información a través de los descubridores de información y motores de búsqueda. A su vez, las BDA varían según la misión, visión y objetivos de la institución a la que pertenecen.

\section{bibliotecas}


En términos generales, según Chowdhury y Chowdhury (2003, p. 8) una BDA tiene las siguientes características:

- Contiene una amplia variedad de recursos digitales de información: textos, imágenes, audios y videos.

- Reduce en gran medida la necesidad de un espacio físico para construir y dar mantenimiento a las bibliotecas digitales.

- Los usuarios están distribuidos por el mundo y, en algunos casos, los diferentes niveles de servicios tienen que ser diseñados para satisfacer las necesidades de los usuarios locales, así como de los remotos.

- Proporciona acceso a diversos tipos de recursos de información, que pueden residir en diferentes servidores alrededor del mundo y, por lo tanto, la infraestructura e interoperabilidad y son puntos muy importantes para el desarrollo y gestión de una biblioteca digital.

- Ofrece variados niveles de acceso a sus recursos, dependiendo de la tipología de usuarios, es decir, hay un nivel de acceso para estudiantes, diferente del de investigadores y profesores.

- Varios usuarios pueden utilizar un recurso de información a la vez, lo que no es posible en una biblioteca tradicional.

- Ha provocado un cambio de modelo no solo en la manera de utilizar la información (de forma impresa a digital), sino también el concepto de propiedad. La mayoría de las bibliotecas digitales proporcionan acceso a materiales que no poseen; algunos de ellos pueden estar disponibles de manera gratuita o bien efectuando un pago para obtener su acceso.

- Con el propósito de cumplir el sueño de construir una infraestructura de información verdaderamente global, las bibliotecas digitales deben ser capaces de manejar recursos de información multilingües.

- Permite una mejor e inmediata búsqueda y recuperación de información.

- La información digital puede ser consultada y utilizada por diferentes personas conforme a sus necesidades individuales.

- Rompe las barreas de tiempo, espacio e idioma. Idealmente, usuarios de cualquier parte del mundo son capaces de utilizar la biblioteca digital en cualquier hora y posiblemente en cualquier idioma.

La BDA posee componentes básicos y complementarios. Antes de abordarlos es preciso señalar que la BDA puede concebirse desde dos perspectivas, tal y como señala Torres Vargas (2008, p. 20):

- Como parte de la biblioteca convencional. En este caso la biblioteca digital permite un uso inmediato de los documentos, la consulta a distancia por parte de los usuarios y la consulta 
de un documento digitalizado por muchos usuarios a la vez. En general, soluciona numerosos problemas u obstáculos que existen en la biblioteca convencional puesto que está pensada para potenciar los servicios de esta; en este sentido, se observa que el desarrollo de bibliotecas digitales se hace bajo el cobijo de bibliotecas nacionales, ya que entre las ventajas se encuentra la conservación de materiales valiosos o poco conocidos.

- Como un sistema autónomo. Es un sistema diferente del que representa la biblioteca convencional. Vista así, la biblioteca digital muestra rasgos y problemáticas propias. En razón de que no se desarrolla en el marco de la biblioteca convencional, no resuelve los obstáculos de aquella, sino que demanda el análisis de las variables que la constituyen y que se relacionan, entre otras cosas, con el desarrollo de colecciones y su organización, el análisis documental, la recuperación y el acceso a la información digital, y con la situación que presenta la BD frente a la educación a distancia. Es por eso que los proyectos para el desarrollo de bibliotecas digitales muestran diferentes características, ya que por lo general reflejan alguna de estas perspectivas. Sin embargo, desde una concepción personal, el segundo enfoque pareciera poseer bases más sólidas porque toma en cuenta el estudio de los fenómenos que se asocian con el manejo de la información digital y atiende de manera más fiel la solución de tales problemas; mientras que al considerar a la biblioteca digital como parte de la biblioteca convencional se está forzando el tratamiento y uso de la información digital bajo el contexto de los impresos.

Los servicios de la BDA están mayormente enfocados a la búsqueda, recuperación, acceso y análisis de los recursos de información de la biblioteca. Actualmente, los procesos de investigación utilizan herramientas y medios de las tecnologías digitales disponibles en la web. Dentro de la literatura especializada este proceso es conocido como e-ciencia y demanda grandes cantidades de información para mantenerse en desarrollo lo que a su vez requiere de otras formas de servicios. En este estudio se han identificado los siguientes servicios digitales disponibles en la BDA:

- Catálogos en línea

- Descubridores de información

- Servicio de acceso remoto a colecciones digitales (a través de la generación de claves de acceso)

- Servicio de información especializada (enfocada al desarrollo de investigaciones académicas o científicas)

- Video tutoriales (que abordan diversos aspectos del proceso de búsqueda y acceso a la información en la BDA)

- Servicio de referencia en línea (proporcionado a través de salas de chat o correo electrónico)

- Renovación de préstamos de recursos de información impresos

- En el caso de los servicios para la búsqueda y acceso a la información disponibles en la BDA, es 
posible identificar la integración de aplicaciones para compartir información bibliográfica en redes sociales. Esta información se encuentra relacionada con un determinado recurso, o bien con diversos contenidos digitales de la BDA.

\section{Web Semántica}

La World Wide Web fue ideada como un recurso de información y comunicación en línea, creada por Tim Berners-Lee para los físicos del Centro Europeo de Investigación Nuclear (siglas en inglés CERN), ubicado en Suiza. El proyecto inicial tenía por objetivo desarrollar una herramienta que facilitara la comunicación interna de los diferentes miembros del CERN, generalmente diseminados en varios países. Por ese entonces, solo se pensaba manejar información textual, sin considerar gráficos, sonidos, ni video. A fines de 1990, el primer software prototipo de la web estuvo terminado y lentamente la tecnología empezó a ser conocida en la comunidad Internet.

El desarrollo de la web ha pasado por una serie de etapas. En la literatura especializada se menciona la evolución de la web basada en diversas generaciones. La web es solo una de las formas de distribuir información a través de Internet, que ha sufrido grandes modificaciones estructurales, tecnológicas, filosóficas y sociales desde que fue creada. Con base en estas transformaciones se ha clasificado su evolución en tres etapas denominadas web 1.0, 2.0 y 3.0.

Las características conceptuales de cada etapa de la web son presentadas a partir de las aportaciones desarrolladas por Tim O'Reilly en su artículo What is Web 2.0: Design Patterns and Business Models for the Next Generation of Software publicado en el 2005. En este trabajo, se menciona que el modelo de la web 1.0 se limita a un espacio de publicación de contenidos corporativos y de servicios, sin participación abierta ni gratuidad en contenidos o servicios de alta relevancia. Las comunidades se formaban fundamentalmente a partir de la oferta de servicios, prescindiendo de espacios para que los miembros publicaran contenidos. Es decir, los usuarios fueron relevantes en tanto eran consumidores.

A su vez, la Web 2.0 es el término dado para describir a la segunda generación de la Web que se centra en la capacidad tecnológica para que los usuarios puedan acceder a espacios de colaboración y para compartir información en línea. Se refiere a la transición de la web estática a una web dinámica, más organizada, basada en el servicio de aplicaciones. Esta generación refleja la transformación del usuario en productor de información, la comunicación determinada por un mayor énfasis a las comunidades compuestas por los propios usuarios y el intercambio de información entre ellos. En este sentido, blogs, wikis y redes sociales son vistos como los componentes característicos de la web 2.0.

Finalmente, la posibilidad de generar una web semántica o también llamada web 3.0 está basada en la idea de añadir metadatos semánticos y ontológicos a la información que posibiliten un mayor éxito en la búsqueda a través de la exploración y en reducción de la dispersión de los resultados. Por ello, muchos de los próximos desarrollos contribuirán a consolidar esta web semántica, y esta, a

\section{bibliotecas}


su vez, permitirá estructurar la información de la manera más similar posible a como los humanos almacenan datos en el cerebro (a través de mapas cognitivos). Se desarrollan nuevos sistemas de interoperabilidad que permitan interpretar metadatos para adaptarse a las acciones de los usuarios.

La web semántica es una web extendida y dotada de mayor significado, apoyada en lenguajes universales, lo cual permite que los usuarios puedan encontrar respuestas de forma más rápida y sencilla, gracias a una información mejor definida. Con esta web, los usuarios podrán delegar tareas en el software que será capaz de procesar el contenido de la información, razonar con este, combinarlo y realizar deducciones lógicas para resolver automáticamente problemas cotidianos (W3C, 2013). Al dotar a la Web de más significado y, por lo tanto, de más semántica, se pueden obtener soluciones a problemas habituales en la búsqueda y recuperación de información, debido a la utilización de una infraestructura común, mediante la cual es posible compartir, procesar y transferir información de forma sencilla.

La web 3.0 se apoya en lenguajes y normas universales que resuelven los problemas ocasionados por una web carente de semántica en la que, en ocasiones, el acceso a la información se convierte en una tarea difícil y frustrante para los usuarios. En este sentido, la aplicación de las tecnologías de la web semántica en la BDA propiciará la conformación de una infraestructura de información con significado que haga posible realizar mejores búsquedas de información a los usuarios.

La web ha sufrido una serie de cambios, en ella se han descubierto nuevas formas de comunicación, de hacer negocios y de realizar trabajo. La comunicación con el mundo en cualquier momento y a bajo coste es posible hoy en día a través de las aplicaciones de la web. Se realizan transacciones económicas a través de Internet y se tiene acceso a millones de recursos, independientemente de la situación geográfica e idiomática. Todos estos factores y muchos otros, han contribuido al éxito de la web. Sin embargo, al mismo tiempo, estos factores que han propiciado su éxito, también han generado sus principales problemas, como la sobrecarga de información y heterogeneidad de fuentes de información con el consiguiente inconveniente de la interoperabilidad, ya que el problema de reunir sistemas de información heterogéneos y distribuidos se conoce como el problema de interoperabilidad, es decir, interoperabilidad es la capacidad de dos o más sistemas o componentes para intercambiar la información y utilizarla (Castañeda de León, 2004. p. 3).

La web semántica trae consigo una serie de innovaciones que aceleran la búsqueda, la recuperación y el intercambio de información en la Web. Antoniou y Van Harmelen (2008) han identificado estas innovaciones en aspectos como la gestión del conocimiento en la web, la creación acelerada de negocios de consumo, el desarrollo de agentes personales y la utilización de ontologías para organizar el conocimiento que se encuentra disponible en la web a través de sus recursos (información textual, visual, sonora, audiovisual, entre otras).

Los elementos esenciales que hacen posible la visión de una web semántica son la estructura de los documentos web basada en lenguaje XML, la descripción de estos documentos con la norma RDF y la relación de estos con el lenguaje de ontologías web (OWL). Con estos elementos, la web

\section{bibliotecas}


semántica busca dar sentido a los contenidos que se publican en este entorno, para ello se pretende facilitar a las computadoras la comprensión de lo que los usuarios de la Web buscan representar en sus contenidos, además de permitir la conexión de los recursos de la Web y extender los hiperenlaces resultantes a relaciones semánticas. Es decir, se busca crear un espacio de información con significado, que entienda las demandas de información de los usuarios, y en el cual la computadora solo sea la herramienta o el medio para llegar a la información disponible en la web.

La web semántica es el resultado de la evolución natural de la web actual, en la que los datos son presentados en un formato único capaz de ser procesado por las computadoras. En este sentido, el World Wide Web Consortium (W3C) ha trabajado en la última década en la definición de diversos estándares, muchos de los cuales han sido utilizados en el desarrollo de múltiples aplicaciones. Aun así, el estado actual de la web presenta un conjunto de limitaciones a las que es deseable encontrarle una solución práctica. Algunas de estas limitaciones son: interoperabilidad, integración, búsqueda y recuperación de información confiable.

La web semántica ha dejado de ser un concepto abstracto para materializarse en diversos proyectos, tanto en la academia como en la industria, con un alto valor para el progreso social. En la última década, la comunidad científica se ha concentrado en el desarrollo de tecnologías y estándares que den dinámica al crecimiento de la web semántica, permitiendo así la construcción de una web dotada de mucho más significado, el cual puede ser procesado tanto por los humanos como por las computadoras.

Los motores de búsqueda semánticos han comenzado a emerger como una de las aplicaciones más prometedoras de las tecnologías de la web semántica, proporcionando una solución eficiente a las limitaciones presentes en los motores de búsquedas tradicionales en los que las consultas son basadas en palabras clave, por lo que no tienen en cuenta la semántica de la necesidad de información expresada. Teniendo en cuenta las aplicaciones de la web semántica se puede apreciar que los problemas de la web actual han sido parcialmente resueltos en algunos ámbitos de aplicación.

Los recursos de información presentes en las BDA podrán ser descritos, organizados y enlazados mediante la adopción de modelos semánticos en sus catálogos de autoridades y bibliográficos. Estos catálogos son una gran fuente de información que puede ser de utilidad en la generación de servicios innovadores de investigación en estas bibliotecas. La web semántica ayuda a resolver algunos de los principales problemas del usuario, delegando sus tareas al software. Gracias a la semántica en la web, el software es capaz de procesar su contenido, razonar con este, combinarlo y realizar deducciones lógicas para resolver problemas cotidianos automáticamente.

El enriquecimiento de las tecnologías de la web semántica se refleja de forma más clara en el área de servicios de información. Morales del Castillo (2011) menciona que en la biblioteca digital las tecnologías de la web semántica se aplican para el desarrollo de servicios de valor añadido, en la definición de una descripción enriquecida, en el establecimiento de ontologías que favorecen la interoperabilidad de los recursos, el desarrollo de interfaces de usuarios, mejoras en la navegación,

\section{bibliotecas}


visualización y presentación de contenidos, entre otras cuestiones. Berners Lee, Hendler y Lassila (2001) conceptualizaron a la web semántica como una extensión de la web actual, en la cual la información está dotada de un significado definido y puede ser procesada por las máquinas e interpretada por los humanos, lo que permite una mejor cooperación entre ambos elementos.

\section{Innovación en los servicios bibliotecarios y de información}

La innovación en los servicios bibliotecarios y de información es un tema que se ha manejado desde la aplicación de las tecnologías de la información y comunicación en las bibliotecas. Este tipo de tecnologías han provocado el surgimiento de nuevos productos y servicios de información en las bibliotecas académicas, pero también han contribuido a la aparición de nuevos procesos informativos relacionados con el uso de información digital. La innovación se considera como sinónimo de producir, asimilar y explotar con éxito una idea, en las esferas económicas y sociales, de forma que aporte soluciones inéditas a los problemas y permita así responder a las necesidades de las personas $y$ de la sociedad (INNOVATEC, 2001). Como parte de la integración de las BDA en el contexto de la web semántica, en la actualidad se hace énfasis en la innovación digital aplicada a los servicios bibliotecarios y de información.

La innovación digital en bibliotecas está caracterizada por el uso de aparatos tecnológicos como computadoras y dispositivos móviles para acceder a la información disponible en las bibliotecas. La idea de la presencia de las BDA en el contexto de la web semántica debe considerar que la web semántica es un espacio digital, con herramientas y lenguajes computacionales que tienen el objetivo de proporcionar un significado concreto a toda la información mediante el establecimiento de relaciones conceptuales, documentales, terminológicas y autorales. La innovación de los servicios bibliotecarios y de información puede definirse en las BDA a través de la utilización y explotación de las herramientas de la web semántica. Dichas herramientas pueden aplicarse en los servicios de la siguiente manera: creación de registros de metadatos RDF, desarrollo de interfaces SPARQL, implementación de servicios semánticos móviles de información y utilización de ontologías y folksonomías para la descripción temática de los recursos de información (ver Figura 1).

La integración de las herramientas de la web semántica pone de manifiesto el dominio de una serie de factores técnicos, procedimentales y funcionales, sobre todo aquellos que tienen que ver con los aspectos estructurales de una BDA. La aplicación de los principios de la web semántica en los servicios bibliotecarios y de información de las bibliotecas digitales académicas están destinados a satisfacer demandas de información complejas y comprender los nuevos comportamientos informativos de los usuarios, sobre todo si se considera que en el entorno digital es sumamente complejo identificar demandas de información y el comportamiento informativo del usuario.

\section{bibliotecas}


Figura 1

Integración de elementos de la web semántica y la Biblioteca Digital Académica

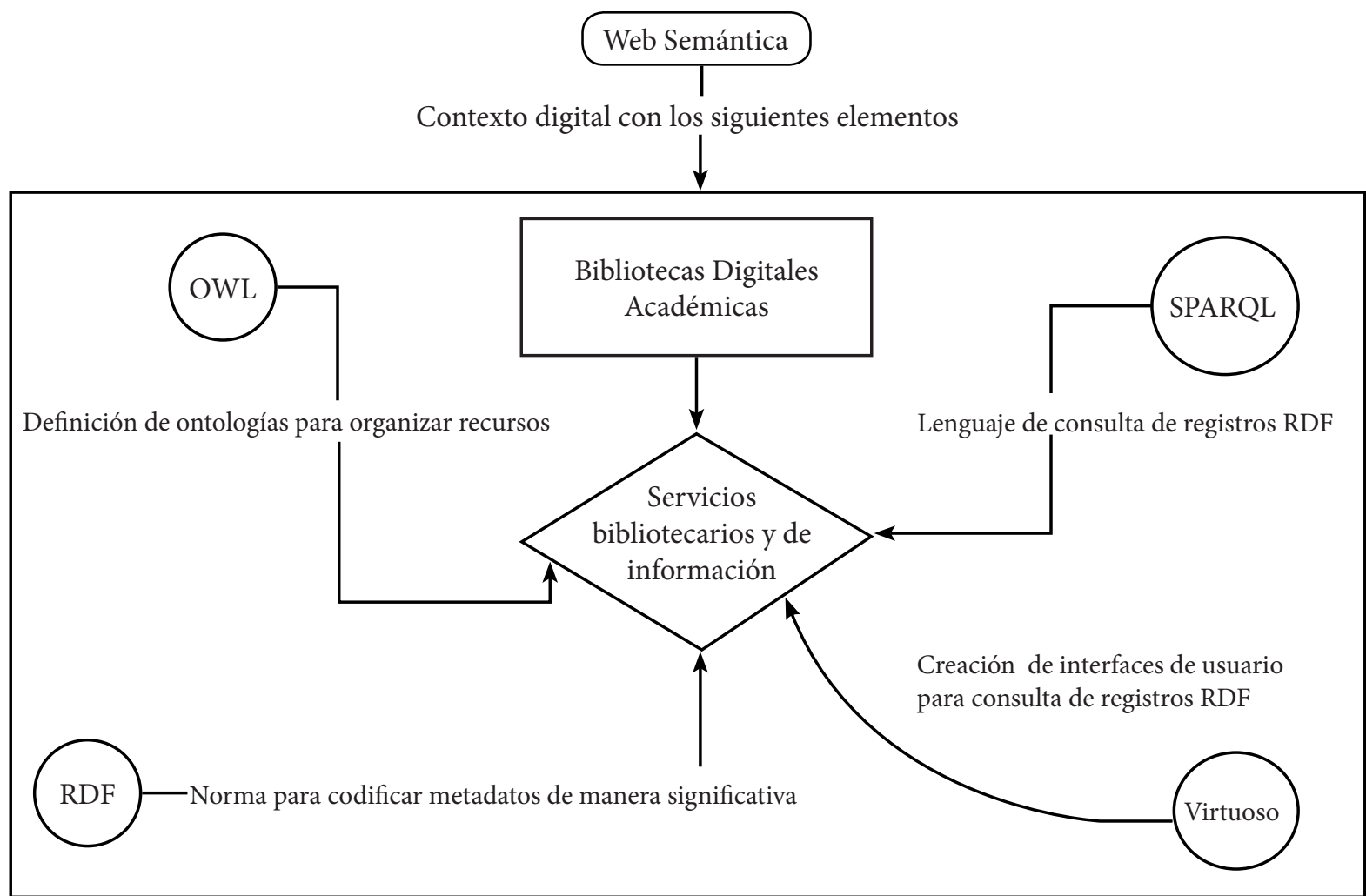

Fuente: elaboración propia de autor, 2015.

\section{Consideraciones finales}

El panorama actual de la integración de las BDA en la web semántica permite apreciar la generación de innovadores servicios bibliotecarios y de información, destinados a atender las demandas informativas de las comunidades de usuarios remotos de las bibliotecas de instituciones de educación superior. Los servicios de metadatos enlazados son un ejemplo de esta innovación, ya que en la actualidad se requiere de amplios sistemas que arrojen una búsqueda de información ampliada y relacionada. En este sentido, los usuarios remotos cobran notable relevancia en este tipo de servicios, ya que es a través del medio digital y mediante el uso de dispositivos tecnológicos que buscan acceder a la información que necesitan y muchas ocasiones lo hacen de manera autónoma.

La presencia de las BDA en la web semántica aporta elementos significativos para la construcción de innovadores servicios bibliotecarios y de información en este tipo de bibliotecas. Algunos de estos

\section{bibliotecas}


servicios poseen valor agregado y son destinados a la búsqueda y recuperación de información de una manera significativa para el usuario. Las tendencias futuras de la aplicación de las herramientas de la Web semántica en los servicios bibliotecarios y de información de las BDA serán aquellas relacionadas con la utilización de normas, principios y tecnologías semánticas para la organización y análisis de la información disponible en bibliotecas. Asimismo, con mayor frecuencia se implementarán interfaces de búsqueda y recuperación de información que permitan obtener un contexto general de la relación significativa entre documentos, es decir que las interfaces tengan la posibilidad de visualizar el universo documental que se relaciona con un recurso de información específico.

\section{Referencias}

Antoniou, G y Van Harmelen, F. (2008). A semantic web premier. MIT: Estados Unidos.

Berners Lee, T., Hendler, J y Lassila, O. (2001). The semantic web: a new form of web content that is meaningful to computers will unleash a revolution of new possibilities. The scientific American, 284 (5).

Castañeda de León, L.M. (2004). Interoperabilidad; estándares. Revista Digital Universitaria, 5 (10): 1-8.

Chowdhury, G. y Chowdhury, S (2003). Introduction to digital libraries. London: Facet Publishing.

INNOVATEC (2001). La Innovación: un factor clave para la competitividad de las empresas. Madrid: CEIM.

O'Reilly, T. (2005). What is Web 2.0: Design Patterns and Business Models for the Next Generation of Software. [Artículo en línea] Disponible en: http://www.oreilly.com/pub/a/web2/archive/whatis-web-20.html

Ortega y Gasset, J (2007). Misión de la universidad. España: Biblioteca Nueva.

Morales Del Castillo, J. (2011). Hacia la biblioteca digital semántica. Gijón: TREA.

Torres, G. (2008). El estudio de la biblioteca digital académica en México mediante el uso de redes sociales. Investigación bibliotecológica, 22 (46): 41-57.

Torres, G (2008). Un modelo integral de biblioteca digital. México: UNAM: Centro Universitario de Investigaciones Bibliotecológicas.

W3C (2015). Guía breve de la web semántica. España. [Artículo en línea] Disponible en http://www.w3c. es/Divulgacion/GuiasBreves/WebSemantica

\section{Nota sobre el Autor}

\footnotetext{
* Mtro. Eder Ávila Barrientos: Estudiante del Doctorado en Bibliotecología y Estudios de la Información en el Posgrado en Bibliotecología y Estudios de la Información. UNAM / FFYL / IIBI. Correo electrónico: eavila86@gmail.com
} 